\title{
Active Decentralised Control Approach for Neutral Current Elimination in Smart Grids
}

\author{
Manish Mohandas ${ }^{1}$, Arun Sebastin ${ }^{2}$ \\ ${ }^{1}$ Student, Saintgits College of Engineering, Kottukulam Hills, Kottayam, India \\ ${ }^{1}$ Assistant Professor, Saintgits College of Engineering, Kottukulam Hills, Kottayam, India
}

\begin{abstract}
In a three phase four-wire system, load imbalance and associated neutral currents pose major problems such as over-heating of rotating machines, malfunctioning of control equipment and protection relays, overloading of neutral conductor in the distribution system and transformer core saturation. As such the neutral current has to be eliminated for maintaining the system reliability and for power quality improvement. So this work proposes an active decentralised control scheme for neutral current elimination by locally providing the necessary compensation using a solar compensator and thereby to improve system stability. The smart grid modelling and analysis are done by time domain simulations in PSCAD software.
\end{abstract}

Keywords: Distribution static compensator (DSTATCOM), Model predictive controller (MPC), power quality (PQ), and Voltage-source inverter

\section{Introduction}

The present and increasing penetration of distributed generation sources (DGs) can facilitate effective decentralized neutral current control in grid networks. A literature review on the various decentralized voltage-control strategies using OLTC, DG, and compensator devices were presented. In general, the decentralized voltage-control schemes do not require a communication infrastructure and, thus, achieve a faster voltage response without the concern of communication failure or time delays. In an economical decentralized voltage control and reactive power compensation scheme, which relies on mechanically switched capacitor banks in the distribution network, is presented. Decentralized voltage-control schemes which rely on compensator devices, such as static var compensator (SVC) and STATCOM, were proposed to achieve faster voltage response and consequently improve the voltage stability and transient response. Furthermore, with the increasing penetration of DG, a decentralized voltage-control scheme using inverter-based DGs is presented for enhancing the steady-state voltage regulation and improving the transient response. In addition to the inverter-based DG voltage regulator, further voltage control is achieved through the diesel synchronous machine excitation control. In, a new hierarchical distributed control structure is introduced by splitting the network into a number of areas that are denoted as "local controllable zone (LCZ)." The DGs and compensator devices in each zone are used to provide the local voltage support without influencing the voltage at external buses in the network.

A Coordinated voltage and reactive power control using master slave approach is discussed in paper[1].An optimal tracking secondary voltage control method developed to achieve effective voltage regulation, enhance the network voltage profile and provide optimal reactive power compensation to the interconnected power system is discussed in paper [2]. A multiagent-based dispatching scheme for distributed generators for voltage support on distribution feeders using control net protocol is presented in paper [3]. A power factor control voltage control (PFC-VC) technique is used for the decentralized voltage control for active distribution networks [4].The enhancement of microgrid dynamic voltage stability using microgrid voltage stabilizer (MGVS) is presented in paper [5]. The implementation issues associated with a novel damping control algorithm for a STATCOM a series compensated Wind Park for mitigating SSR and damping power system oscillations is discussed in paper [6]. The voltage stability, reactive power reserves, fault ride through stability, steady state performance of smart grids are discussed in paper[7]. In these research papers, effective reactive power compensation schemes and the impact of neutral current and its elimination are not discussed.

In this paper, the neutral current due to phase imbalancing is eliminated using an active decentralized control approach and is implemented on a smartgrid environment using simulations on P-SCAD.

\section{Test System}

In a centralized approach for coordinating the various compensating devices, such as shunt capacitors, shunt reactors, and voltage regulators has been proposed to mitigate the impacts of DG on the system voltage. The problem is solved optimally using genetic algorithms, where the amount of operation of each compensating device is determined such that losses and voltage deviation are minimized . A smart-grid centralized approach, where DG provides voltage control via a secure communication infrastructure, is proposed in. Sensitivity analysis was carried out to find the locations where reactive power has a greater impact with an aim to regulate the voltage to their specified values. In , a GA-based procedure is used to adjust the optimal setting of the OLTC and all shunt switching capacitors based on load forecast. This method aims at minimizing the power loss and improve the voltage profile. A multiagent system was also proposed to determine the optimal reactive power support from DG as well as optimal tap setting of OLTC for voltage control. Furthermore, a 


\section{International Journal of Science and Research (IJSR) \\ ISSN (Online): 2319-7064}

Index Copernicus Value (2013): 6.14 | Impact Factor (2014): 5.611

centralized reactive power control scheme for grid interface inverters was proposed in to optimize the reactive power within the system. Centralized approaches typically require significant investments in sensors, communications, and dedicated controllers which make such solutions costly. A distributed control scheme, using a multiagent system (MAS) approach, that relies on coordinating the voltage regulator with the available DGs by determining the optimal DG reactive power output to maintain the voltage during emergency conditions was proposed in certain papers. A DG-based optimal distributed algorithm was proposed in that relies on decomposing the system into subnetworks, thus reducing the need for global communication. These proposed control methods require an efficient communication system connecting all buses (whether they are DG buses or not). Further, controlling all buses will lead to a computational burden in large distribution systems. In addition, the possibility of managing the different responses of DGs, conventional generation, and OLTC for maximizing the dynamic reactive power reserve in the system has not been investigated. This paper introduces a novel coordinated voltage-control scheme for smart grids with OLTCs and DGs that relies on a dynamic master/slave control approach. The scheme relies on adaptively changing the roles (master or slave) based on three states of operation, of the devices (inverter-based DG, and online tap changer) within the smart grid, depending on system conditions. Voltage and reactive power constraints are used to determine the master/slave operation of each device. The system is designed to provide slow and medium speed response, using low-pass filters, for the OLTC and diesel generator and fast response for VSCDGs. Therefore, it achieves different bandwidth control dedicated by the decentralized voltage controllers and the reactive power-management scheme. A comprehensive simulation study demonstrates the operation and performance of the proposed control scheme, which includes improved voltage profile, better fault-ride through (FRT) performance, and increased transient stability margins for at the wind turbine terminals. Additional compensation, to maintain the bus voltage close to 1 p.u., is provided by a STATCOM (with a 3\% droop setting) located at the wind turbine. The system also includes a utility-scale (4.2 MW) PV power system connected to the distribution system. The $\mathrm{PV}$ arrays are connected to theMV bus via a $0.69 / 25-\mathrm{kV}$ transformer as shown in Fig. 1. The distribution system loads are a mix of static and dynamic loads including induction motors. The distribution system is connected to the transmission equivalent grid through a $25 / 132-\mathrm{kV}$ substation transformer. The CVC scheme coordinates, in an indirect way, the reactive power among the OLTC, VSC-DGs (PV and WTGs).

\section{Base System Representation}

The distribution power system Is illustrated in fig. 1. The distribution system has DG sources at buses b5 and b6, which includes, utility-scale photovoltaic (PV) plant, and wind turbine generators (WTGS), respectively. A doubly fed induction generator (DFIGS) is implemented for the wtgs.. The wtgs are connected to the medium-voltage bus via a $0.69 / 25$-kvtransformer. The reactive power absorbed by the DFIG is partly compensated by capacitor banks connected at the wind turbine terminals.
The PSCAD model of the induction machine is represented by a wound rotor type which can be operated in either "speed control" mode or "torque control" mode. The induction machine initially starts in the speed control mode to reach the rated per-unit speed and is then switched to torque control mode. The rotor terminals are available externally to connect external components, such as resistances and other circuits, to modify the characteristics to allow speed control. Additional compensation, to maintain the bus voltage close to 1 p.u., is provided by a statcom (with a $3 \%$ droop setting) located at the wind turbine. The developed time-domain model of the OLTC consists of the tap ratio as a discrete variable, which can be varied within 16 steps and each step is adjusted to 0.00625 p.u.. The PV plant is composed of 40 inverters with a total capacity of $4.2 \mathrm{MW}$. The grid-side converter (GSC) is modeled as a controllable voltage source

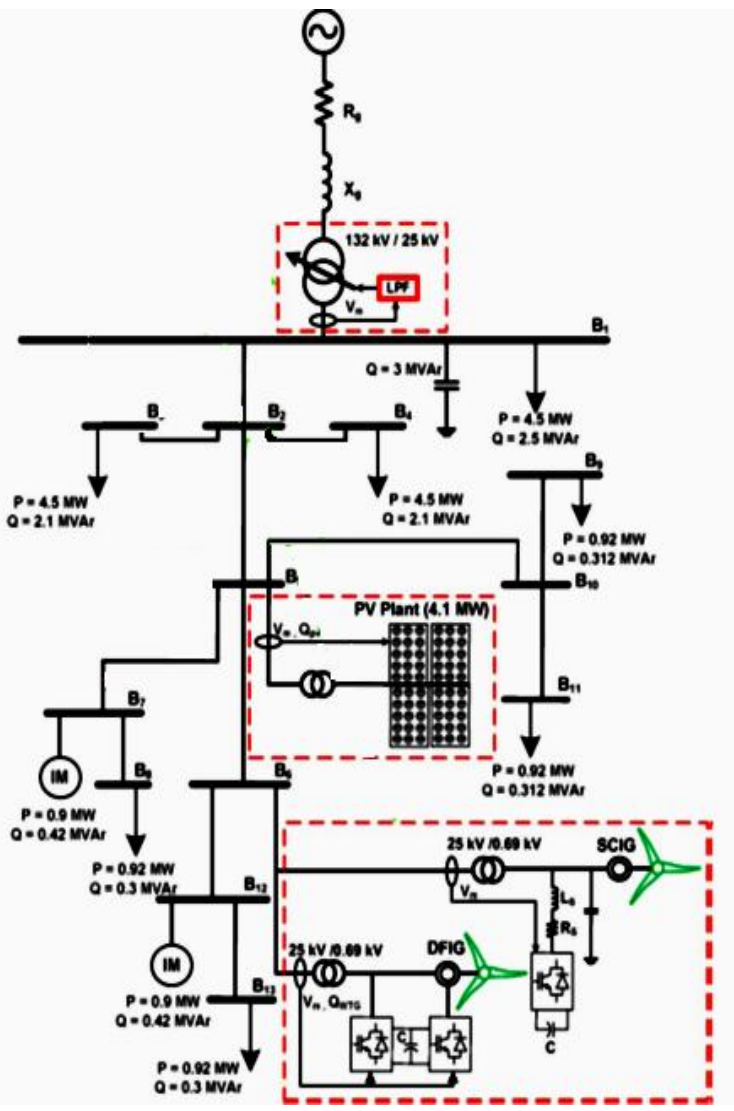

Figure 1: Distribution test system comprised of a PV power system, WTGs, connected to a grid.

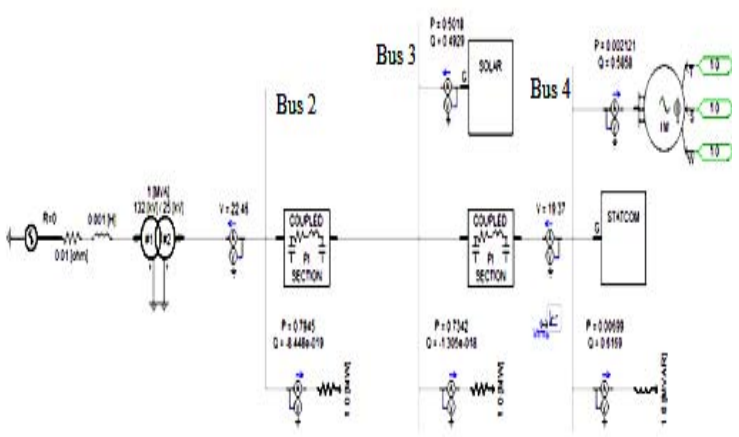

Figure 2: Base System model in PSCAD 


\section{International Journal of Science and Research (IJSR) \\ ISSN (Online): 2319-7064}

Index Copernicus Value (2013): 6.14 | Impact Factor (2014): 5.611

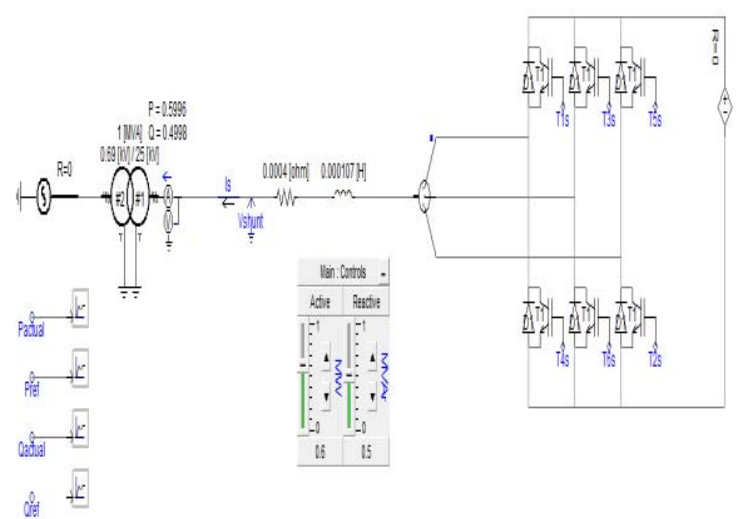

Figure 3: Solar inverter model in PSCAD

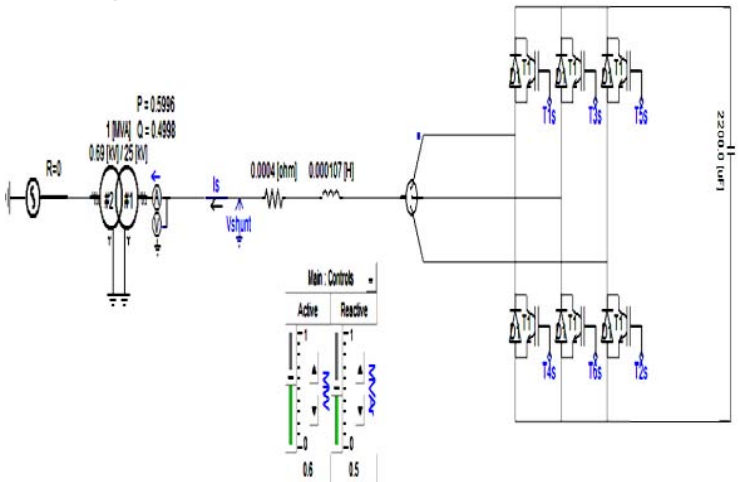

Figure 4: Statcom model in PSCAD

To determine the effects of neutral current due to phase imbalancing, we incorporate an unbalanced load into the test system at bus 3 . The unbalanced load modelled in the paper is represented by unequal resistive loads as shown in fig 5 . The presence of an unbalanced load in the system introduces a phase imbalance and thereby a neutral current in the system. The existence of neutral current in the system causes over-heating of rotating machines, malfunctioning of control equipments and protection relays, overloading of neutral conductor in the distribution system and transformer core saturation. So, the neutral current has to be eliminated for maintaining the system reliability.

In this paper, a control scheme is implemented to eliminate the neutral current due to phase imbalance. An active decentralised control scheme is used in which the neutral current is detected and nullified by a current of equal and opposite magnitude, known as anti-phase current, supplied by the solar inverter. Here the solar inverter has the capability to provide the reactive power. The control scheme and the generation of anti-phase current is shown in the fig 6

\section{Simulation Result}

The simulation results for balanced and unbalanced condition are shown in the following figures. It can be seen that after some transient oscillations the active and reactive power at bus 3 comes to be a stable value after 4 secs.Also, the reactive power supplied by the solar inverter stabilizes to a value after $4 \mathrm{sec}$. The active and reactive power

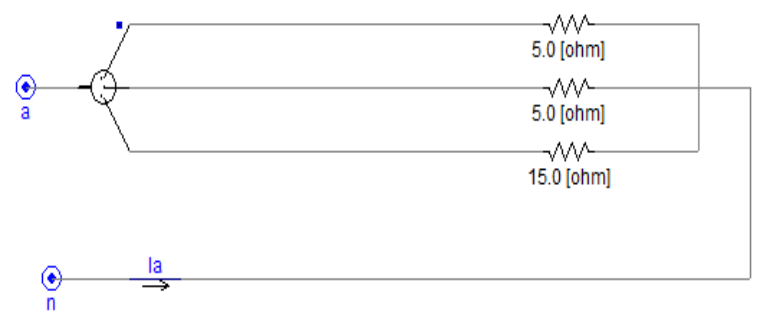

Figure 5: Representation of unbalanced load

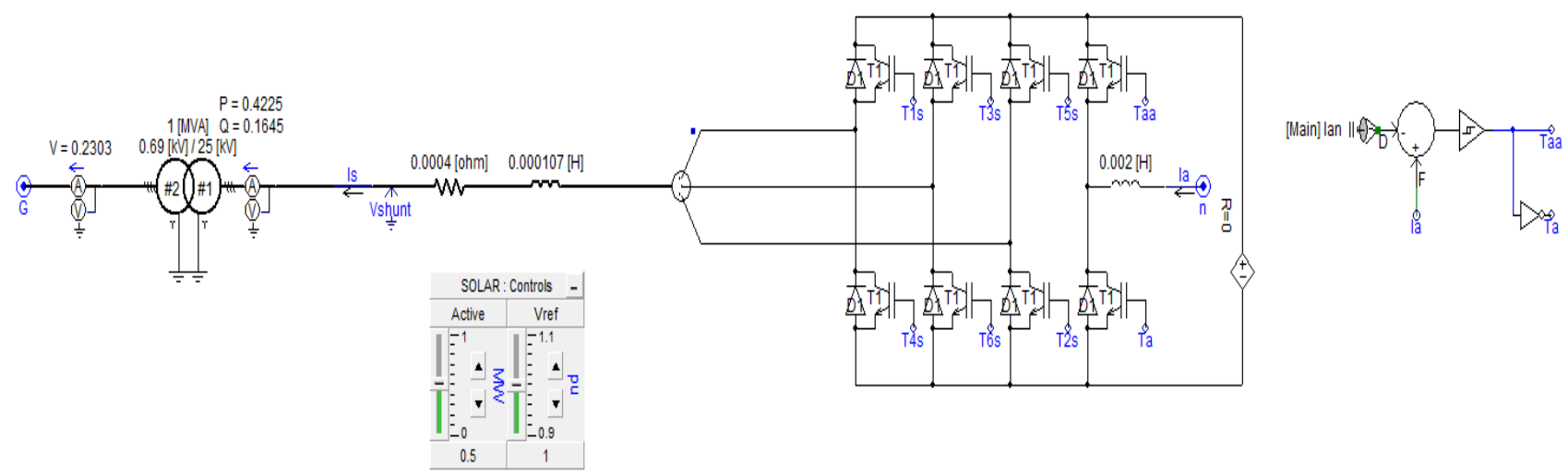

Figure 6: Neutral current compensation and its control 


\section{International Journal of Science and Research (IJSR) \\ ISSN (Online): 2319-7064}

Index Copernicus Value (2013): 6.14 | Impact Factor (2014): 5.611

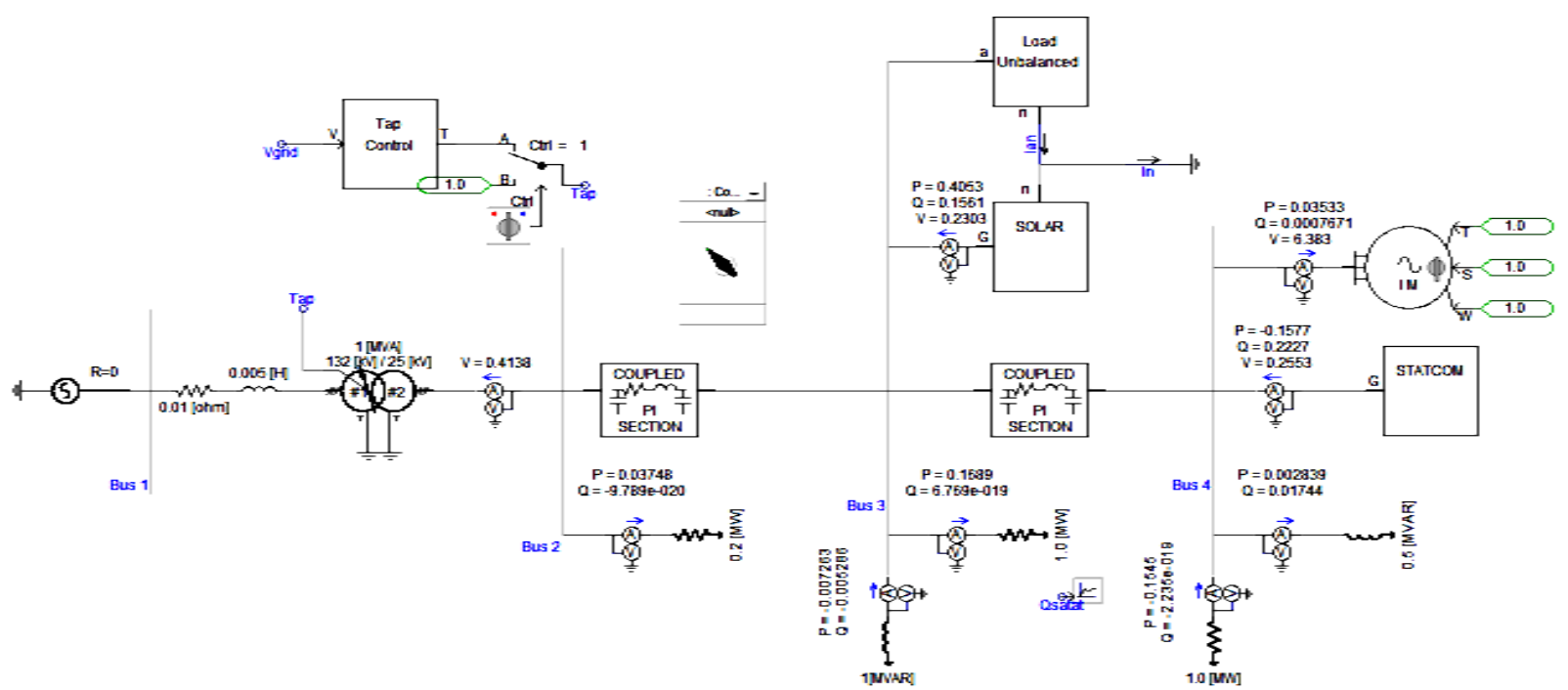

Figure 7: Representation of complete system model with control scheme

efore load imbalance

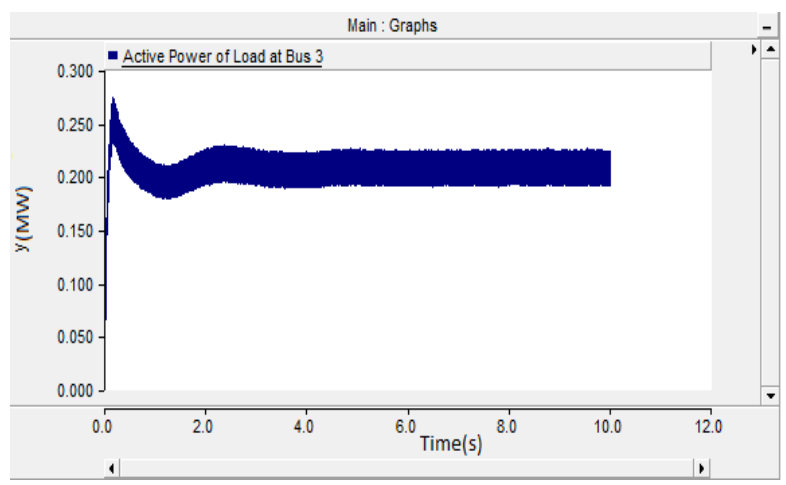

Figure 8: Active power of load at bus 3

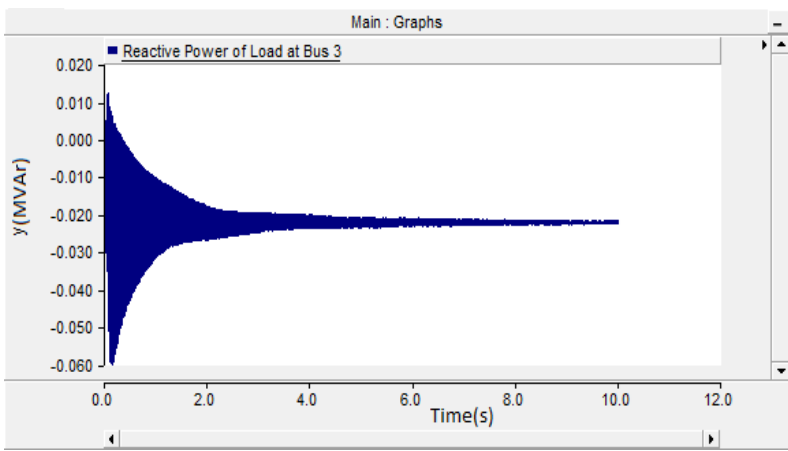

Figure 9: Reactive power of load at bus 3

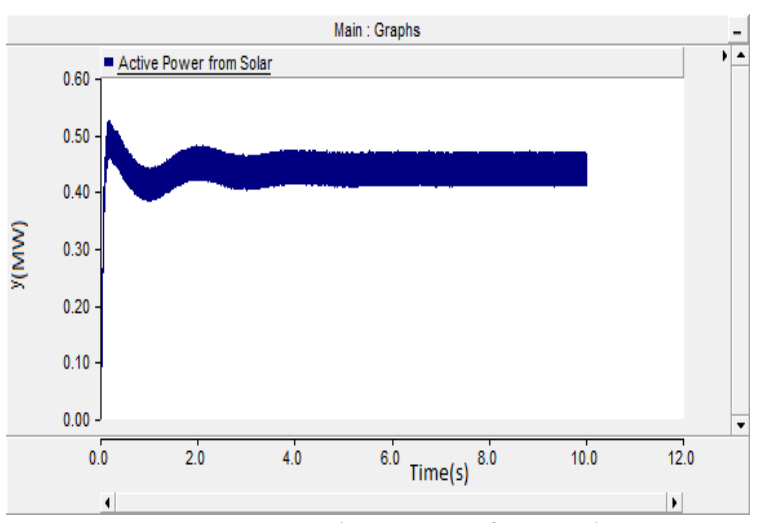

Figure 10: Active power from solar

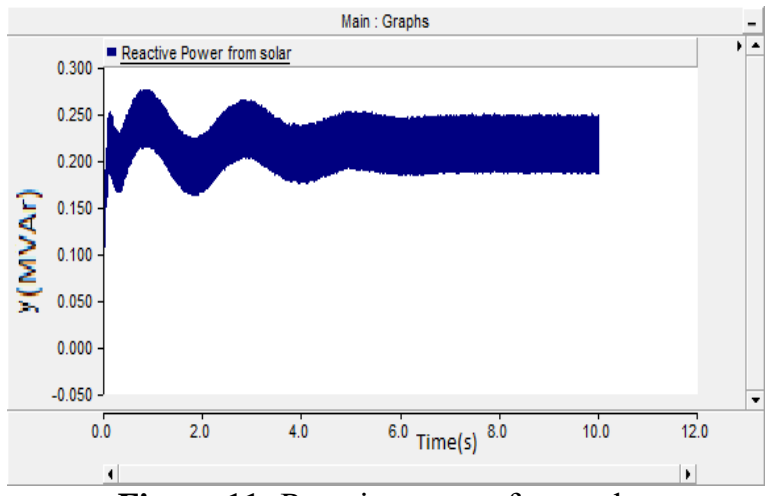

Figure 11: Reactive power from solar

The neutral current produced by the unbalanced load is shown in fig12.It exists throughout the system as far as the presence of unbalanced load. The magnitude of neutral current, $\mathrm{I}_{\mathrm{an}}$ obtained during the simulation of the test system is $10 \mathrm{kA}$. This is depicted in fig 12 .

The anti-phase current generated by the solar compensator, $I_{a}$ is shown in fig 13. With the help of this anti-phase current, the neutral current in the system can be eliminated as. First, the neutral current generated by the unbalanced load is detected by the control scheme incorporated in the solar inverter. As soon as the neutral current is detected, it triggers the thyristors $\mathrm{T}_{\mathrm{a}}$ and $\mathrm{T}_{\mathrm{aa}}$ shown in fig 6 . This produces the anti-phase current to compensate the system neutral current. As such a little or no neutral current flows through the neutral conductor thereby eliminating the hazards of the neutral current in the system. The resulting neutral current through the neutral conductor is shown in fig 14. It can be seen from fig 14 that the resulting neutral current has value almost equal to zero. 


\section{International Journal of Science and Research (IJSR) \\ ISSN (Online): 2319-7064}

Index Copernicus Value (2013): 6.14 | Impact Factor (2014): 5.611

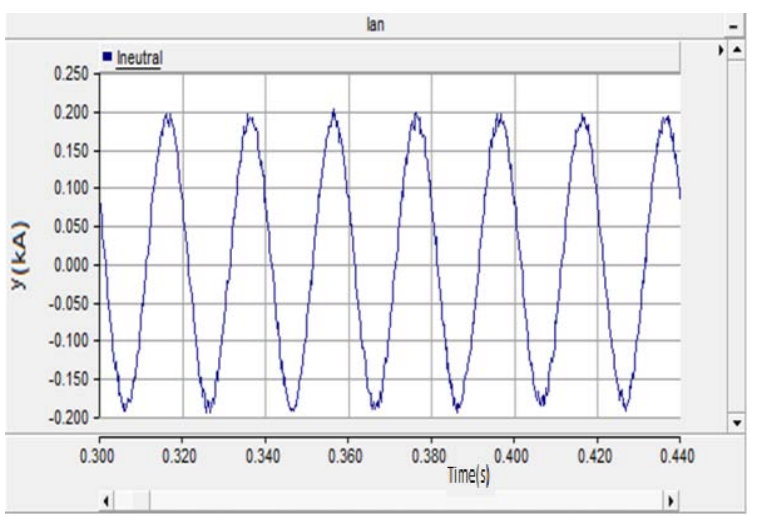

Figure 12: Neutral current from unbalanced load

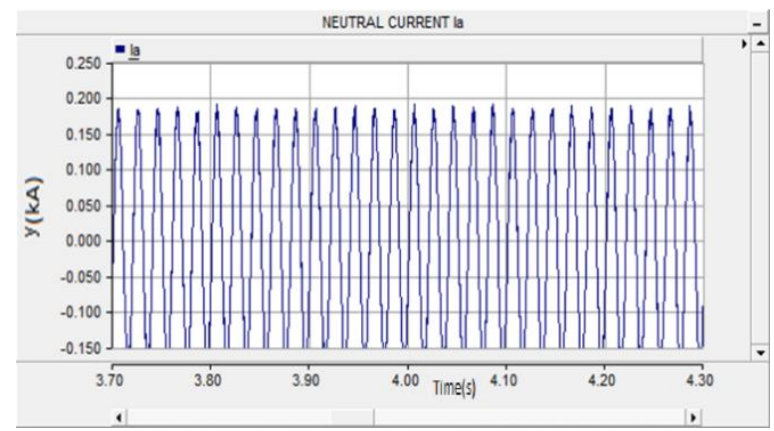

Figure 13: Neutral current generated by compensator

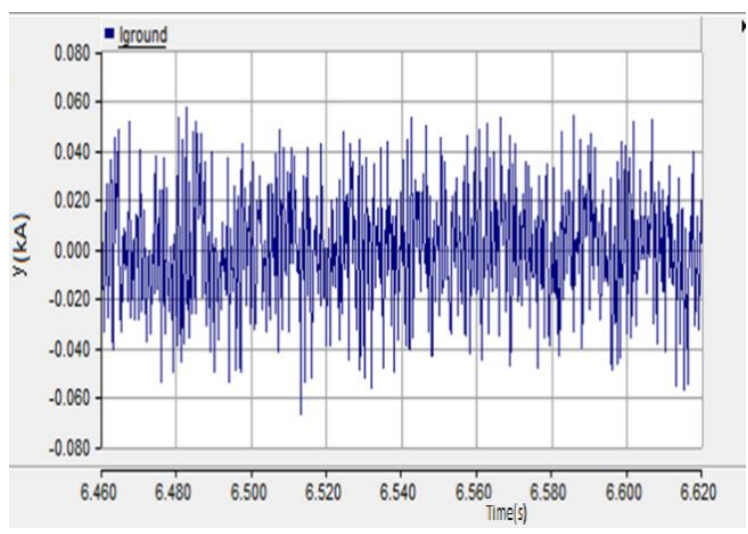

Figure 14: Final neutral current

\section{Conclusion}

A grid model was prepared using PSCAD. Neutral current were observed during insertion of an unbalanced load. An effective Neutral current elimination method as proposed was tested and simulated and this reduces the neutral current in the system almost by $80 \%$.Voltage profile was found to be more stable and FRT capability increased after compensation. Protection schemes coordinated with this active decentralised neutral current elimination method can be practically implemented.

\section{References}

[1] F. A. Viawan and D. Karlsson, "Coordinated voltage and reactive power control in the presence of distributed generation," in Proc. IEEE Power Energy Soc. Gen. Meeting-Convers. Del. Elect. Energy 21st Century, Jul. 20-24, 2008, pp. 1-6.
[2] M. El Moursi, G. Joos, and C. Abbey, "A secondary voltage control strategy for transmission level interconnection of wind generation,"

[3] M. E. Baran and I. M. El-Markabi, "A multiagent-based dispatching scheme for distributed generators for voltage support on distribution feeders,".

[4] A. Tamersi, G. Radman, and M. Aghazadeh, "Enhancement of microgrid dynamic voltage stabilty using microgrid voltage stabilizer," Proc. IEEE Southeastcon, pp. 368-373, Mar. 17-20, 2011.

[5] P. N. Vovos, A. E. Kiprakis, A. R. Wallace, and G. P. Harrison, "Centralized and distributed voltage control: Impact on distributed generation penetration," IEEE Trans. Power Syst., vol. 22, no. 1, pp. 476-483, Feb. 2007.

[6] L. Yu, D. Czarkowski, and F. de Leon, "Optimal distributed voltage regulation for secondary networks with DGs," IEEE Trans. Smart Grid, vol. 3, no. 2, pp. 959-967, Jun. 2012.

[7] T. Sansawatt, L. F. Ochoa, and G. P. Harrison, "Decentralized voltage and thermal management to enable more distributed generation connection," in Proc. CIRED Workshop, Lyon, France, 2010, pp. 1-4.

[8] M. E. Baran and I. M. El-Markabi, "A multiagent-based dispatching scheme for distributed generators for voltage support on distribution feeders," IEEE Trans. Power Syst., vol. 22, no. 1, pp. 52-59, Feb. 2007.

[9] M. S. El-Moursi, B. Bak-Jensen, and M. H. AbdelRahman, "Novel STATCOM controller for mitigating SSR and damping power system oscillations in a series compensated wind park,"

[10] Aarti Gupta, Dinesh Jain, SurenderDahiya, "Neutral current compensation techniques in autonomous windenergy sources”, Int J Energy Environ Eng (2014). 